Article

\title{
Prophylactic Anti-Cytomegalovirus Hyperimmunoglobulin in Critically Ill Liver Transplant Patients: Impact on Early Immunology and Survival
}

\author{
Arno Kornberg ${ }^{1, *(\mathbb{D})}$, Ulrike Witt ${ }^{1}$, Jennifer Kornberg ${ }^{2}$, Katharina Müller ${ }^{3}$, Helmut Friess ${ }^{1}$ and \\ Katharina Thrum ${ }^{4}$ \\ 1 School of Medicine, Department of Surgery, Klinikum rechts der Isar, Technical University of Munich, \\ 81675 Munich, Germany; Ulrike.Witt@tum.de (U.W.); Helmut.Friess@tum.de (H.F.) \\ 2 Department of Anaesthesiology, Klinikum Großhadern, Ludwig-Maximilian-University of Munich, \\ 81377 Munich, Germany; JenniferKornberg@aol.com \\ 3 Department of Surgery, Friedrich-Schiller-University of Jena, 07747 Jena, Germany; \\ katharina.mueller86@gmx.de \\ 4 Institute of Pathology, Helios Klinikum Berlin, 14165 Berlin, Germany; katharina.thrum@gmail.com \\ * Correspondence: ArnoKornberg@aol.com; Tel.: +49-89-4140-5087; Fax: +49-89-4140-4870
}

Received: 31 January 2020; Accepted: 28 February 2020; Published: 29 February 2020

\begin{abstract}
Background: Anti-cytomegalovirus hyperimmunoglobulin (CMVIg) was shown to provide beneficial immunodulatory properties beyond antiviral efficacies. The aim of this retrospective study was to assess the impact of prophylactic CMVIg treatment on early outcome following liver transplantation (LT) in critically ill patients. Methods: Forty-three cirrhotic patients requiring pre-LT intensive care due to multiorgan failure were analyzed. Twenty-eight patients with enhanced CMV risk $(\mathrm{D}+/ \mathrm{R}+; \mathrm{D}+/ \mathrm{R}-; \mathrm{D}-/ \mathrm{R}+)$ received prophylactic CMVIg for a minimum of 7 days, while 15 patients $(\mathrm{D}-/ \mathrm{R}-)$ did not. Results: Post-transplantation rates of intra-abdominal infections $(28 \%$ vs. $61.1 \% ; p=0.03)$, Epstein-Barr virus infections ( $0 \%$ vs. $33.3 \% ; p=0.034)$, allograft rejections $(0 \%$ vs. $22.2 \% ; p=0.013)$ and sepsis-related mortality ( $4 \%$ vs. $27.8 \% ; p=0.026)$ were significantly lower, whereas incidence of CMV infections ( $4 \%$ vs. $22.2 \% ; p=0.066)$ tended to be lower in the CMVIg subset. In multivariate analysis, only pretransplant elevated serum lactate level (hazard ratio $=34.63 ; p=0.009$ ) and absence of CMVIg therapy (hazard ratio $=21.76 ; p=0.023$ ) were identified as independent promoters of 3-month mortality. Conclusion: Prophylactic treatment with CMVIg reduces predisposition for severe immunological and septic events and, thereby, early mortality in critically ill liver recipients.
\end{abstract}

Keywords: liver transplantation; critical illness; multiorgan failure; CMV infection; anti-CMV hyperimmunoglobulin; immune modulation

\section{Introduction}

The implementation of the "sickest first" allocation policy using the so-called Model of End-Stage Liver Disease (MELD) score almost 2 decades ago has clearly proven effective in reducing waiting list mortality in many large liver transplantation (LT) programs around the world [1]. Due to a growing disparity between demand and availability of appropriate donor organs, pre-LT waiting times and final MELD scores have been, however, significantly increasing in recent years; consequently, transplant patients have become sicker and more complex [1,2]. In particular, multiorgan failure (MOF) requiring intensive care unit (ICU) support, as is common in patients with acute-on-chronic 
liver failure (ACLF), was shown to be a major risk factor of early morbidity and mortality following LT [3-5]. Considering other established transplant indications that are associated with a much better prognosis, LT in ICU-bound patients not only represents a medical challenge but also raises important ethical issues [6]. In order to avoid futility in patients being "too sick" for transplant, a profound interdisciplinary risk assessment using reliable prognostic factors is mandatory [7]. Nonetheless, owing to an enhanced susceptibility to severe immunological and septic complications, the postoperative course in this specific LT-subset remains highly demanding [3-5], thus requiring the implementation of effective immunoprotective concepts beyond adjustment of immunosuppressive treatment [8,9].

In this context, prevention of early cytomegalovirus (CMV) infection seems to be of particular prognostic importance, since besides causing direct organ damages, it may indirectly affect outcome by modulating the recipients' immune response. Triggered by proinflammatory cytokine release and various immunosuppressive mechanisms, an immunological risk may, thereby, be aggravated [10]. Although antiviral prophylaxis by ganciclovir or valganciclovir is nowadays widely established in patients with high-risk CMV immunoglobulin (Ig) G donor (D)/recipient (R) seroconstellation $(\mathrm{D}+/ \mathrm{R}-)$, this is usually not applicable in severely immunocompromised allograft recipients due to intolerance against common side effects like renal dysfunction, leukopenia and thrombocytopenia [11]. That is why anti-CMV hyperimmunoglobulin (CMVIg) for passive immunization is meanwhile increasingly reconsidered in selected patients [12]. Although tolerability and antiviral efficacies of CMVIg have clearly been confirmed in the past [13], its use is currently not a recommended standard for antiviral prophylaxis or treatment in the LT setting [14]. In recent years, there seems to be growing suggestive evidence that, apart from antiviral activities, CMVIg may provide anti-inflammatory and immunoregulatory properties that could be valuable to counteract CMV-related immune reactions [12,15-17]. We hypothesized that critically ill liver recipients, who are particularly threatened by early inflammatory events, might benefit from these positive immunomodulatory capabilities. The aim of the presented retrospective study was to assess the prognostic impact of prophylactic CMVIg therapy on early outcome in a series of highest acuity ICU-bound LT patients.

\section{Material and Methods}

\subsection{Study Cohort}

From a prospectively managed LT database, 43 adult cirrhotic patients with hepatic decompensation requiring ICU support prior to LT were identified between 2007 and 2012. At the time of listing, all of them had expressed their consent that data may be used for scientific purpose. The study was conducted in accordance with the Declaration of Helsinki, and the protocol was approved by the institutional review board of the Medical School of the Technical University of Munich (No. 217/15). It is noteworthy that no organs from executed donors were used in this investigation. Progressive liver cirrhosis was assessed by clinical and radiographic criteria prior to registration and could be confirmed on histopathological analysis of the explanted livers. Patients with acute liver failure were not included in the study. In addition to liver dysfunction, all patients suffered from associated neurologic, hemodynamic, respiratory or renal failure requiring mechanical invasive ventilation, continuous vasopressor administration or renal replacement therapy. "Fatal triad" indicated the necessity of pulmonary, circulatory and renal support. Laboratory (lab.) MELD scores at listing and at LT were used for describing MELD dynamics ( $\triangle$ MELD score $=($ lab.) MELD score at registration (lab.) MELD score at LT). Diagnosis and grading (grades 1-3) of ACLF and related MOF were based on the European Association for the Study of the Liver-Chronic Liver Failure (EASL-CLIF) Consortium consensus criteria. The respective CLIF-C organ failure (OF) score (range: 11-18) at LT was calculated for each patient [18]. Preoperative serum lactate levels (normal range: $<1.8 \mathrm{mmol} / \mathrm{L}$ ) were used for describing severity of critical illness and tissue hypoxia $[19,20]$, whereas values of C-reactive protein (CRP; normal range: $<0.5 \mathrm{mg} / \mathrm{dL}$ ), interleukin-6 (IL-6; normal range: $<7 \mathrm{pg} / \mathrm{mL}$ ) and procalcitonin 
(PCT; normal range: $<0.1 \mathrm{ng} / \mathrm{mL}$ ) reflected extent of systemic inflammatory response reaction prior to LT [21].

\subsection{Donor Factors}

The following prognostically relevant donor factors were included in risk analysis: age, body mass index, donor risk index, liver allograft reperfusion performance, cold and warm ischemia times.

\subsection{Treatment with CMVIg and Post-Transplant Follow-Up}

According to our standard transplant protocol, ICU-bound liver recipients with an increased CMV risk ( $\mathrm{D}+/ \mathrm{R}+; \mathrm{D}+/ \mathrm{R}-; \mathrm{D}-/ \mathrm{R}+)$ received prophylactic intravenous CMVIg (Cytotect ${ }^{\mathrm{TM}}$, Biotest, Dreieich, Germany) with a dose of $100 \mathrm{IE}$ (Ehrlich)/ $\mathrm{kg} /$ day ( $\hat{=} 1 \mathrm{~mL} / \mathrm{kg} /$ day; infusion rate: $0.8 \mathrm{~mL} / \mathrm{kg} / \mathrm{h}$ ), starting at the first post-LT day and continued for a minimum of 1 week, whereas $\mathrm{D}-/ \mathrm{R}-$ patients were not treated. As a result of MOF, none of the study patients were eligible for specific antiviral medication. Serum DNA loads of CMV and Epstein-Barr virus (EBV) were determined in all patients twice a week during post-LT ICU stay and weekly at minimum thereafter for the first 3 months by real-time PCR (ThermoFisher 7500 Real-Time PCR System, ThermoFisher Scientific Inc., USA). Detectable CMV DNAemia at any level indicated CMV infection. Immunosuppressive therapy consisted of a dual regimen by tacrolimus (Tac; intended trough level: $5-7 \mathrm{ng} / \mathrm{dL}$ ) and corticosteroids. Biopsy-proven allograft rejection was treated with methylprednisolone bolus/Tac-dose adjustment. Peak serum levels of CRP, IL-6 and PCT during 1 month post-LT described early systemic state of inflammation [21].

\subsection{Statistical Analysis}

Quantitative variables were expressed in median and range and compared by ANOVA analysis. Qualitative variables were reported in frequencies and percentages and compared by chi-squared test. Post-LT survival rates were determined by Kaplan-Meier method and compared using log rank test. Factors of early (3-month) mortality were assessed by uni- and multivariate logistic regression analysis. A $p<0.05$ was considered statistically significant. All statistical analyses were performed using SPSS 24.0 software (IBM Inc., Munich, Germany).

\section{Results}

\subsection{Patients' Characteristics}

Median age of the study population was 58 years (range: 32-68), with ethyltoxic liver disease (58.1\%) and viral hepatitis (23.3\%) being the major transplant indications. Median (lab.) MELD scores at listing and at LT were 28 (range: 7-40) and 38 (range: 30-40), respectively, resulting in a median $\triangle$ MELD of 9 (range: 0-32). Pre-LT waiting times measured from registration and from final hospital admittance ranged between 4 days and 36 months (median: 3 months) and between 3 and 71 days (median: 15 days), respectively. All patients were ICU-bound prior to LT for a median of 6 days (range: 2-42). With regard to ACLF, patients were classified grade 1 in $2(4.7 \%)$, grade 2 in $10(23.3 \%)$, and grade 3 in 31 cases $(72.1 \%)$. Twenty-five patients required dialysis $(58.1 \%)$, 36 patients received vasopressors $(83.7 \%)$, and 18 patients $(41.9 \%)$ were on ventilation treatment prior to LT. Twenty-five liver recipients $(58.1 \%)$ received prophylactic anti-CMV Ig for a median of 7 days (range: 7-20; Table 1). Recipients' age was significantly lower and pre-LT CRP and IL-6 levels tended to be lower in the CMVIg subset, whereas in contrast, no significant differences were noticed regarding severity of liver disease and MOF. In addition, donor variables were comparable between both subsets (Table 1). 
Table 1. Pretransplant patients' and donors' characteristics.

\begin{tabular}{|c|c|c|c|}
\hline Patients' Characteristics & Non-CMVIg $(n=18)$ & CMVIg $(n=25)$ & $p$ Value \\
\hline Age & $62(39-68)$ & $56(32-67)$ & 0.007 \\
\hline Male sex & $12(66.7 \%)$ & $16(64 \%)$ & 0.856 \\
\hline \multicolumn{4}{|c|}{ Genesis of Cirrhosis } \\
\hline Ethyltoxic & $12(66,6 \%)$ & $13(52 \%)$ & \multirow{5}{*}{0.519} \\
\hline Viral hepatitis & $4(22.2 \%)$ & $6(24 \%)$ & \\
\hline Autoimmune & $1(5.6 \%)$ & $5(20 \%)$ & \\
\hline Cryptogenic & $1(5.6 \%)$ & $0(0 \%)$ & \\
\hline Other & $0(0 \%)$ & $1(4 \%)$ & \\
\hline \multicolumn{4}{|c|}{ CMV Seroconstellation } \\
\hline$D-/ R-$ & $18(100 \%)$ & $0(0 \%)$ & \multirow{4}{*}{$<0.001$} \\
\hline$D-/ R+$ & $0(0 \%)$ & $7(28 \%)$ & \\
\hline$D+/ R-$ & $0(0 \%)$ & $14(56 \%)$ & \\
\hline$D+/ R+$ & $0(0 \%)$ & $4(16 \%)$ & \\
\hline Serum bilirubin level in $\mathrm{mg} / \mathrm{dL}$ at LT & $14(4.8-31.9)$ & $14(6.9-58)$ & 0.232 \\
\hline Serum creatinine level in $\mathrm{mg} / \mathrm{dL}$ at $\mathrm{LT}$ & $2.6(0.5-3.8)$ & $2.5(1.4-5.5)$ & 0.579 \\
\hline International normalized ratio at LT & $2.3(1.5-3.1)$ & $2.5(1.2-4.0)$ & 0.186 \\
\hline Serum natrium level in $\mathrm{mmol} / \mathrm{L}$ at $\mathrm{LT}$ & $130(125-145)$ & $133(119-147)$ & 0.523 \\
\hline (lab.) MELD score at listing & $25.5(12-40)$ & $30(7-40)$ & 0.486 \\
\hline (lab.) MELD score at LT & $39(31-40)$ & $38(30-40)$ & 0.621 \\
\hline$\triangle$ MELD score & $10(0-28)$ & $9(0-32)$ & 0.752 \\
\hline Waiting time from registration in months & $3(0.5-25)$ & $3(0.1-36)$ & 0.642 \\
\hline Waiting time from final admittance in days & $14.5(3-41)$ & $17(3-71)$ & 0.225 \\
\hline Ascites at LT & $18(100 \%)$ & $23(92 \%)$ & 0.219 \\
\hline Bacterial infection at LT & $10(55.6 \%)$ & $10(40 \%)$ & 0.313 \\
\hline Duration of pre-LT ICU stay in days & $7.5(0-35)$ & $4(0-42)$ & 0.276 \\
\hline Ventilation at LT & $9(50 \%)$ & $8(32 \%)$ & 0.234 \\
\hline Vasopressors at LT & $15(83.3 \%)$ & $21(84 \%)$ & 0.953 \\
\hline Renal replacement therapy at LT & $10(55.5 \%)$ & $15(60 \%)$ & 0.771 \\
\hline Fatal triad at LT & $6(33.3 \%)$ & $6(24 \%)$ & 0.501 \\
\hline \multicolumn{4}{|c|}{ Acute on Chronic Liver Failure at LT } \\
\hline Grade 1 & $0(0 \%)$ & $2(8 \%)$ & \multirow{3}{*}{0.452} \\
\hline Grade 2 & $5(27.8 \%)$ & $5(20 \%)$ & \\
\hline Grade 3 & $13(72.2 \%)$ & $18(72 \%)$ & \\
\hline \multicolumn{4}{|c|}{ Organ Failures (According CLIF-C) at LT } \\
\hline Liver & $11(61.1 \%)$ & $13(52 \%)$ & 0.553 \\
\hline Kidney & $18(100 \%)$ & $23(92 \%)$ & 0.219 \\
\hline Circulation & $15(83.3 \%)$ & $21(84 \%)$ & 0.935 \\
\hline Respiration & $10(55.6 \%)$ & $9(36 \%)$ & 0.201 \\
\hline Coagulation & $10(55.6 \%)$ & $14(56 \%)$ & 0.977 \\
\hline Neurology & $9(50 \%)$ & $9(36 \%)$ & 0.359 \\
\hline \multicolumn{4}{|c|}{ Number of Organ Failures at LT } \\
\hline$\leq 3$ & $7(38.9 \%)$ & $13(52 \%)$ & \multirow{2}{*}{0.395} \\
\hline$>3$ & $11(61.1 \%)$ & $12(48 \%)$ & \\
\hline CLIF-C OF score at LT & $16(11-18)$ & $15(11-18)$ & 0.460 \\
\hline Lactate level in $\mathrm{mmol} / \mathrm{L}$ at $\mathrm{LT}$ & $3.0(0.9-10)$ & $3(0.9-5.8)$ & 0.373 \\
\hline CRP level in $\mathrm{mg} / \mathrm{dL}$ at $\mathrm{LT}$ & $3.8(0.7-6.1)$ & $3(0.4-7.2)$ & 0.058 \\
\hline PCT level in $\mathrm{ng} / \mathrm{mL}$ at $\mathrm{LT}$ & $2.5(0.4-7)$ & $1.3(0.3-49.9)$ & 0.480 \\
\hline IL-6 level in $\mathrm{pg} / \mathrm{mL}$ at LT & $22(6-78)$ & $17(5-45)$ & 0.074 \\
\hline \multicolumn{4}{|c|}{ Donor Variables } \\
\hline Age & $54(18-75)$ & $57(29-71)$ & 0.428 \\
\hline Body mass index & $1.8(1.1-2.5)$ & $1.9(1.3-2.3)$ & 0.399 \\
\hline Donor risk index & $26(22-32)$ & $26(19-40)$ & 0.881 \\
\hline \multicolumn{4}{|c|}{ Allograft Reperfusion Performance } \\
\hline Well & $5(27.8 \%)$ & $10(40 \%)$ & \multirow{4}{*}{0.342} \\
\hline Acceptable & $8(44.4 \%)$ & $10(40 \%)$ & \\
\hline Moderate & $5(27.8 \%)$ & $3(12 \%)$ & \\
\hline Poor & $0(0 \%)$ & $2(8 \%)$ & \\
\hline Cold ischemia time in min & $545(350-1140)$ & $600(360-900)$ & 0.857 \\
\hline Warm ischemia time in min & $51(30-68)$ & $45(20-90)$ & 0.596 \\
\hline
\end{tabular}

CMVIg, anti-CMV hyperimmunoglobulin; LT, liver transplantation; CMV, cytomegalovirus; MELD, model of end-stage liver disease; ICU, intensive care unit; ACLF, acute-on-chronic liver failure; CLIF-C, chronic liver failure consortium; OF, organ failure; CRP, C-reactive protein; PCT, procalcitonin; IL-6, interleukin-6. 


\subsection{Early Post-LT Outcome}

Treatment was well-tolerated in all cases, without need of dose reduction. Median total number of CMVIg doses/patient was 7 (range: 7-20). The incidence of intra-abdominal infections was significantly higher in the non-CMVIg cohort, while rates of systemic bacterial infections were comparable (Table 2). One patient of the CMVIg- (4\%) but four of the non-CMVIg subset $(22.2 \%)$ developed CMV infection. Apart from that, incidence of EBV infections was significantly higher without therapy. Allograft rejection rates were $0 \%$ for the CMVIg cohort and $22.2 \%$ for the non-CMVIg cohort (Table 2). Post-transplant peak serum levels of CRP, IL-6 and PCT were all significantly lower following anti-CMV Ig treatment, whereas Tac levels were comparable (Figure 1).

Table 2. Post-LT outcome at 3 months.

\begin{tabular}{lccc}
\hline & Non-CMVIg $(n=\mathbf{1 8})$ & CMVIg $(n=25)$ & $p$ Value \\
\hline Systemic bacterial infection & $7(38.9 \%)$ & $9(36 \%)$ & 0.847 \\
Intra-abdominal bacterial infection & $11(61.1 \%)$ & $7(28 \%)$ & 0.030 \\
CMV infection & $4(22.2 \%)$ & $1(4 \%)$ & 0.066 \\
EBV infection & $3(33.3 \%)$ & $0(0 \%)$ & 0.034 \\
Biopsy proven allograft rejection & $4(22.2 \%)$ & $0(0 \%)$ & 0.005 \\
Mortality & $7(38.9 \%)$ & $2(8 \%)$ & 0.014 \\
Death by septic MOF & $5(27.8 \%)$ & $1(4 \%)$ & 0.026 \\
\hline
\end{tabular}

CMVIg, anti-CMV hyperimmunoglobulin; CMV, cytomegalovirus; EBV, Epstein-Barr virus; MOF, multiorgan failure.
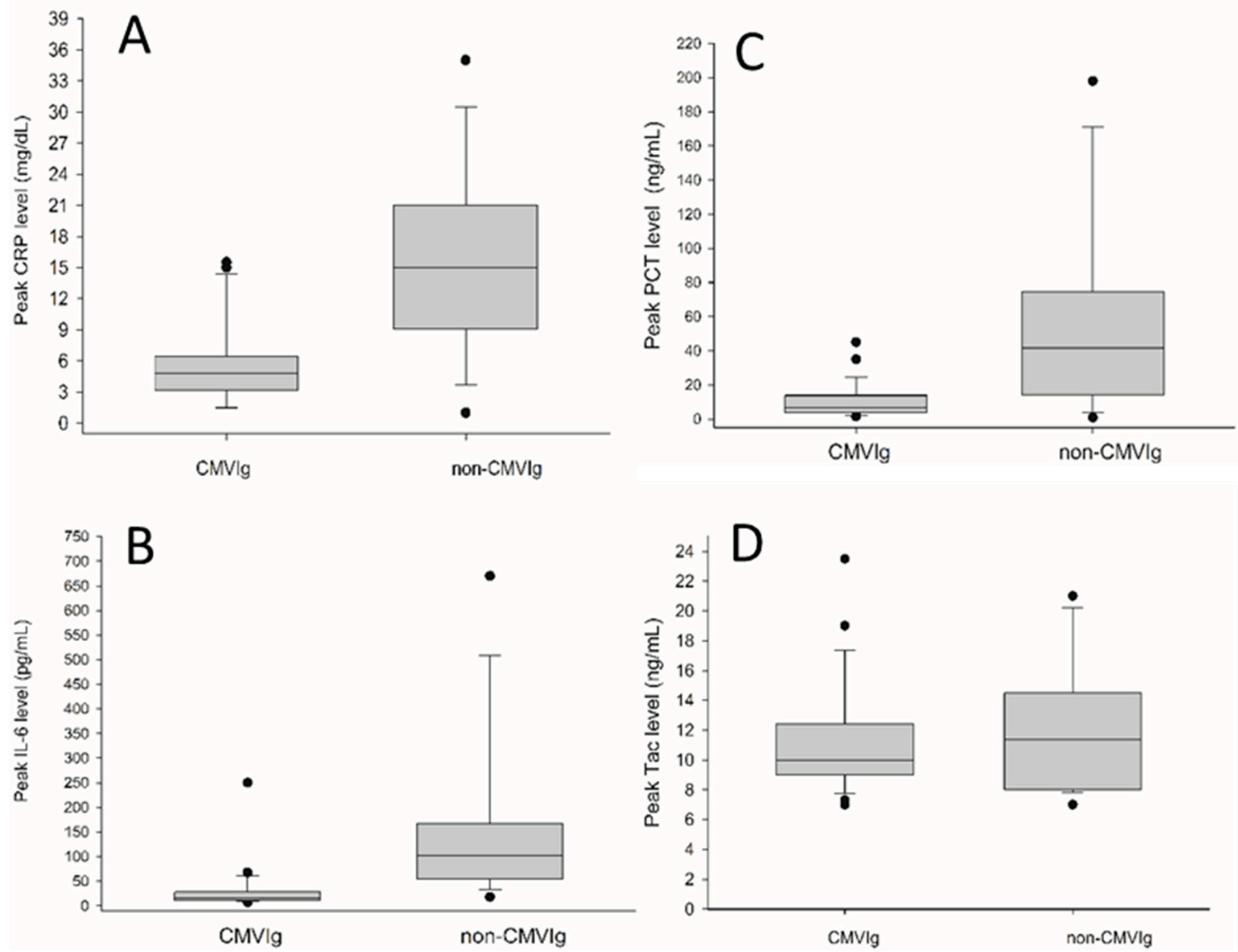

Figure 1. Post-LT peak serum levels of CRP (A), $p<0.001$; IL-6 (B), $p<0.001$ and PCT (C) $p=0.017$ were all significantly lower following CMVIg treatment, whereas peak Tac levels (D) $p=0.336$ were comparable. 


\subsection{Prognostic Factors of 3-Month Mortality}

At 3 months post-LT, nine liver recipients had died (20.9\%), two in the CMVIg (8\%) subgroup and seven in the non-CMVIg subgroup (38.9\%). Corresponding mortality rates due to septic MOF were $4 \%$ and $27.8 \%$, respectively (Table 2). In univariate analysis, MELD score at listing, $\triangle$ MELD, ICU stay, fatal triad, CMVIg treatment, cold ischemia time and serum levels of lactate, CRP and IL-6 exerted a prognostic impact (Table 3). Only pre-LT elevated lactate level and absence of CMVIg therapy were identified as independent promoters of 3-month mortality, whereas cold ischemia time almost reached statistical significance (Table 4). Respective survival curves are illustrated in Figure 2.

Table 3. Univariate prognostic factors of 3-month mortality.

\begin{tabular}{|c|c|c|c|}
\hline Variables & Alive at 3 Months $(n=34)$ & Dead at 3 Months $(n=9)$ & $p$ Value \\
\hline Recipients'Age & $58(32-68)$ & $61(39-67)$ & 0.278 \\
\hline Male sex & $21(61.7 \%)$ & $7(77.8 \%)$ & 0.370 \\
\hline MELD at listing & $30(7-40)$ & $15(12-40)$ & 0.019 \\
\hline MELD at LT & $38(30-40)$ & $40(31-40)$ & 0.582 \\
\hline$\triangle \mathrm{MELD}$ & $9(0-32)$ & $16(0-28)$ & 0.047 \\
\hline Waiting time from registration in months & $2.25(0.1-36)$ & $6(1.5-25)$ & 0.134 \\
\hline Waiting time from final admittance in days & $13.5(3-71)$ & $26(3-43)$ & 0.229 \\
\hline Duration of pre-LT ICU stay in days & $5(2-16)$ & $10(2-42)$ & 0.002 \\
\hline Ascites at LT & $32(94.1 \%)$ & $9(100 \%)$ & 0.456 \\
\hline Bacterial infection at LT & $15(44.1 \%)$ & $5(55.6 \%)$ & 0.541 \\
\hline Grade of ACLF ( 3 vs. 1 or 2 ) at LT & $26(76.5 \%)$ & $5(55.6 \%)$ & 0.214 \\
\hline Number of pre-LT organ failures according CLIF-C & $4(1-6)$ & $5(2-6)$ & 0.579 \\
\hline CLIF-C OF score at LT & $15.5(11-18)$ & $16(11-18)$ & 0.935 \\
\hline Ventilation at LT & $13(38.2 \%)$ & $5(55.6 \%)$ & 0.349 \\
\hline Vasopressors at LT & $29(85.3 \%)$ & $7(77.8 \%)$ & 0.587 \\
\hline Renal replacement therapy at LT & $18(52.9 \%)$ & $7(77.8 \%)$ & 0.179 \\
\hline Fatal triad at LT & $7(20.6 \%)$ & $5(55.6 \%)$ & 0.038 \\
\hline Lactate level in $\mathrm{mmol} / \mathrm{L}$ at LT & $3(0.9-5.6)$ & $4.9(1.9-10)$ & $<0.001$ \\
\hline CRP level in $\mathrm{mg} / \mathrm{dL}$ at $\mathrm{LT}$ & $3(0.4-6)$ & $5.6(0.8-7.2)$ & 0.013 \\
\hline PCT level in $\mathrm{ng} / \mathrm{mL}$ at LT & $1.5(0.3-49.9)$ & $3.6(2-35.5)$ & 0.264 \\
\hline IL-6 level in pg/mL at LT & $19(5-45)$ & $25(6-78)$ & 0.003 \\
\hline Peak Tac level in ng/dL & $10(7-23.5)$ & $14(7-20)$ & 0.169 \\
\hline Lack of CMVIg treatment & $11(32.4 \%)$ & $7(77.8 \%)$ & 0.014 \\
\hline Donors' Age & $57(18-75)$ & $46(27-71)$ & 0.172 \\
\hline Donor body mass index & $1.9(1.07-2.50)$ & $1.62(1.08-2.15)$ & 0.082 \\
\hline Donor risk index & $26(19-40)$ & $25(22-32)$ & 0.447 \\
\hline Moderate/poor allograft reperfusion & $10(29.4 \%)$ & $0(0 \%)$ & 0.063 \\
\hline Cold ischemia time in min & $585(350-900)$ & $720(450-1140)$ & 0.009 \\
\hline Warm ischemia time in min & $51.5(20-70)$ & $40(300-90)$ & 0.681 \\
\hline
\end{tabular}

MELD, model of end-stage liver disease; LT, liver transplantation; ICU, intensive care unit; ACLF, acute-on-chronic liver failure; CLIF-C, chronic liver failure consortium; OF, organ failure; CRP, C-reactive protein; $\mathrm{PCT}$, procalcitonin; IL-6, interleukin-6; CMVIg, anti-CMV hyperimmunoglobulin.

Table 4. Multivariate prognostic factors of 3-month mortality.

\begin{tabular}{lccc}
\hline \multicolumn{1}{c}{ Variables } & HR & CI 95\% & $p$ Value \\
\hline Lactate level (> vs. $\leq 3 \mathrm{mmol} / \mathrm{L})$ & 34.63 & $2.383-503.198$ & 0.009 \\
CMVIg treatment (no vs. yes) & 21.76 & $1.540-307.368$ & 0.023 \\
Cold ischemia time (> vs. $\leq 600 \mathrm{~min})$ & 11.49 & $0.966-136.786$ & 0.053 \\
\hline
\end{tabular}

$\mathrm{HR}$, hazard ratio; $\mathrm{CI}$, confidence interval; CMVIg, anti-CMV hyperimmunoglobulin. 

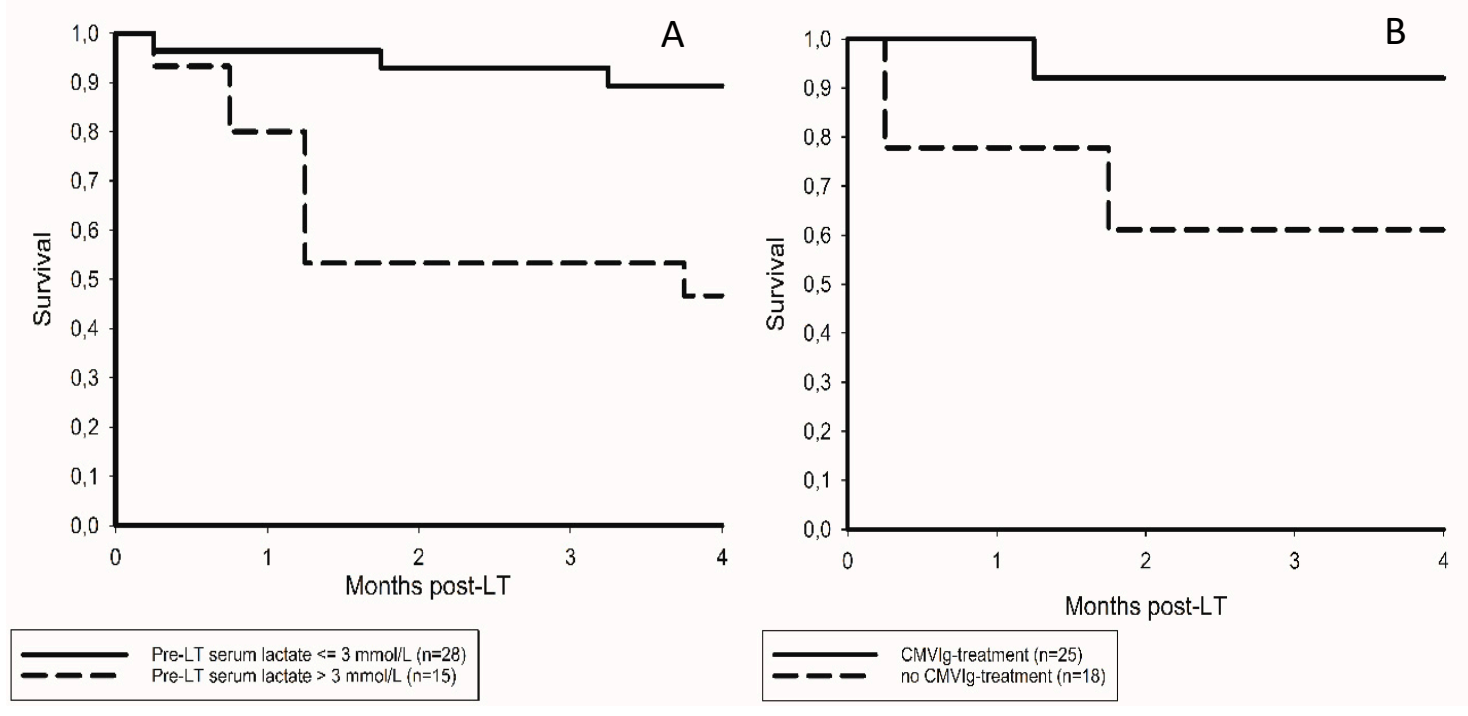

Figure 2. (A) Postoperative survival rates at 3 months were $92.9 \%$ and $53.3 \%$ in patients with pre-LT serum lactate levels $\leq$ versus $>3 \mathrm{mmol} / \mathrm{L}$, respectively, log rank $=0.002$. (B) Survival rates in patients receiving and those not receiving CMVIg were $92 \%$ and $61.1 \%$, respectively, log rank $=0.02$.

\section{Discussion}

The results of our study indicate that prophylactic administration of CMVIg reduces early morbidity and mortality following LT in critically ill cirrhotic patients, most probably related to positive immune modulation with regard to CMV risk, allograft immunogenicity and predisposition for septic events (Table 2).

Owing to evidenced antiviral efficacies and low adverse-effect profile, anti-CMV Ig has for a long time been the cornerstone in prophylaxis and treatment of CMV infection following solid organ transplantation. However, in the context of potent and cheaper antiviral drugs that have been implemented in the last years [11], it has clearly lost significance. Recent guidelines issued by the Transplantation Society do not generally recommend prophylaxis with CMVIg, but rather suggest augmentation of antiviral medication in high-risk organ recipients, who require intensified immunosuppression, such as patients following thoracic and intestinal transplantation. Apart from that, its administration may be considered on an individual basis as rescue treatment in severe CMV diseases or ganciclovir-resistant courses [14].

Currently, there is growing experimental and clinical evidence that, independent from established antiviral capacities, CMVIg provides immunological benefits that seem to be rather comparable to those of polyvalent Ig preparations $[12,15,16]$. Even though the complex mechanisms of immune modulation are not yet fully understood, it appears to affect several aspects of the innate and adaptive immune response, resulting in a combination of immunosuppressive (inhibition of dendritic maturation and suppression of $\mathrm{T}$ cells), immunostimulatory (passive anti-CMV immunization and increasing levels of naïve B cells) and anti-inflammatory (decreasing production of IL-2, interferon-y and IL-10) efficacies [12,15-17,22-24]. In fact, lower incidences of immunological and infectious complications following anti-CMV Ig therapy have been demonstrated in some recent studies on adult and pediatric LT [25-28]. In the largest investigation thus far on this topic presented several years ago, Fischer et al. reported on reduced risk of allograft loss and death in 2350 liver recipients receiving CMVIg, without, however, stratifying data on extent of liver decompensation and MOF [28].

To the best of our knowledge, we here present the first study analyzing the impact of prophylactic CMVIg in severely ill ICU-bound liver transplant patients. Apart from pre-LT serum lactate, which is an established prognostic factor in this clinical setting [19,20], only lack of anti-CMV Ig therapy was 
identified as an independent promoter of early mortality in our series (Table 4). According to our findings, different immunomodulatory aspects may be discussed as possible triggers for the observed prognosis improvement.

First, despite higher serological CMV risk, we noticed a clear trend of lower CMV incidence in the CMVIg subset ( $4 \%$ vs. $22.2 \%$ ), which in view of well-known direct and indirect CMV-related harms could have been involved in superior outcome. Thus, our data emphasize on the prognostic importance of implementing passive CMV-specific immunity in those cases where recommended antiviral prophylaxis is not feasible [17]. Second, rates of acute allograft rejections ( $0 \%$ vs. $27.8 \%)$ and severe septic events (4\% vs. $27.8 \%$ ) were both significantly lower in our treatment group (Table 2$)$, even though contradictory trends are frequently observed in clinical reality. Therefore, anti-CMV Ig appears to stabilize the immunological balance between allograft immunogenicity on the one hand and susceptibility for infections on the other, which may be fundamental for reducing early morbidity and mortality in immunocompromised liver recipients [29]. In this context, a higher rate of EBV reactivation ( $33 \%$ vs. $0 \%$; Table 2 ) may be regarded as another indicator of immunological dysbalance in the non-CMVIg subgroup [30]. Third, significantly lower post-LT peak serum levels of proinflammatory mediators (Figure 1) suggested a beneficial impact on early systemic immunological response reaction [21].

Retrospective design and low sample size are limitations of our study and may well explain the high $95 \%$ confidence interval in our multivariate analysis (Table 4). Apart from that, the prognostic value of CMVIg in low risk patients (D-/R-) remained undefined. Also, nonconsidered differences in proinflammatory activation might have biased our results. In fact, the immunological performance may be influenced by (pre-LT) background inflammation, donor liver function and (post-LT) immunosuppressive treatment [30]. Apart from recipients' age, which in turn may have had a prognostic impact in our analysis [3-5], we did not find significant differences in patients' characteristics, donor features and immunosuppressive levels (Table 1). However, dynamic immune monitoring including repeat IgG level determinations and immunosuppressive drug exposure studies are required for a more precise immunological assessment. Finally, we cannot exclude that treatment with nonspecific Ig might have provided similar immunomodulatory efficacies than CMVIg. Recently, a beneficial efficacy of IgM-enriched Ig on hemodynamic stability and 30-day mortality was reported in a series of 21 patients suffering from post-LT vasoplegia requiring vasopressor treatment. However, there was no control group and, moreover, the study was lacking data on pre-LT status and CMV-specific outcome [31]. Thus, in our opinion, the combination of both anti-CMV impact and immunoregulatory properties renders CMVIg more attractive for critically ill liver recipients than conventional Ig preparations, which do not specifically inhibit viral activities. However, a comparative investigation is needed to further elucidate this issue.

Particular strengths of our investigation included a comprehensive generation of preoperative background and post-LT follow-up data and, above all, topicality of the subject and novelty of our findings. Although the "sickest first" policy has clearly defined and transparent rules and regulations [32], decision-making process in times of a dramatic donor shortage is determined not only by considerations of medical feasibility, but also, to a large extent, by ethical concerns. Individual urgency and possible benefit in highly complex LT candidates have to be weighed against common utility [6], which is hampered by lack of well-defined drop-out criteria and, besides, by an enormous pressure to succeed with regard to survival rates and profitability of the program [6,7]. Instead of defining transplant futility $[7,20,33]$, we were rather focusing on immunological aspects to improve survival of highest acuity LT patients who most probably had been rejected at utility-guided centers. Against this background, our reported 3-month survival rate of $92 \%$ following anti-CMV Ig treatment represents an extraordinary outcome result, especially when considering that death would have been the most likely alternative. 


\section{Conclusions}

In summary, our study suggests that critically ill liver recipients benefit from prophylactic CMVIg treatment by an improved risk profile for immunological and infectious complications and, thereby, from reduced early mortality. However, this needs to be validated in a prospective multicenter study approach, including immunological analyses for clarifying the underlying immunomodulatory mechanisms of action.

Author Contributions: Conceptualization, A.K. and U.W.; Methodology, A.K.; Validation, H.F. and J.K.; Data Curation, A.K. and U.W. and K.T. and K.M.; Writing - Original Draft Preparation, A.K.; Writing - Review \& Editing, H.F. and J.K. All authors have read and agreed to the published version of the manuscript.

Funding: This work was supported by the German Research Foundation (DFG) and the Technical University of Munich within the funding program Open Access Publishing.

Conflicts of Interest: The authors declare no conflicts of interest.

\section{References}

1. Sacleux, S.C.; Samuel, D.A. Critical Review of MELD as a Reliable Tool for Transplant Prioritization. Semin. Liver Dis. 2019, 39, 403-413. [CrossRef] [PubMed]

2. Samuel, D.; Coilly, A. Management of patients with liver diseases on the waiting list for transplantation: A major impact to the success of liver transplantation. BMC Med. 2018, 16, 113. [CrossRef]

3. Sundaram, V.; Jalan, R.; Wu, T.; Volk, M.L.; Asrani, S.K.; Klein, A.S.; Wong, R.J. Factors Associated with Survival of Patients With Severe Acute-On-Chronic Liver Failure Before and After Liver Transplantation. Gastroenterology 2019, 156, 1381-1391. [CrossRef] [PubMed]

4. Thuluvath, P.J.; Thuluvath, A.J.; Hanish, S.; Savva, Y. Liver transplantation in patients with multiple organ failures: Feasibility and outcomes. J. Hepatol. 2018, 69, 1047-1056. [CrossRef] [PubMed]

5. Levesque, E.; Winter, A.; Noorah, Z; Daurès, J.P.; Landais, P.; Feray, C.; Azoulay, D. Impact of acute-on-chronic liver failure on 90-day mortality following a first liver transplantation. Liver Int. 2017, 37, 684-693. [CrossRef] [PubMed]

6. Bobbert, M.; Ganten, T.M. Liver allocation: Urgency of need or prospect of success? Ethical considerations. Clin. Transplant. 2013, 27 (Suppl. 25), 34-39. [CrossRef] [PubMed]

7. Linecker, M.; Krones, T.; Berg, T.; Niemann, C.U.; Steadman, R.H.; Dutkowski, P.; Clavien, P.A.; Busuttil, R.W.; Truog, R.D.; Petrowsky, H. Potentially inappropriate liver transplantation in the era of the "sickest first" policy - A search for the upper limits. J. Hepatol. 2018, 68, 798-813. [CrossRef]

8. Luo, Y.; Ji, W.B.; Duan, W.D.; Shi, X.J.; Zhao, Z.M. Delayed introduction of immunosuppressive regimens in critically ill patients after liver transplantation. Hepatobiliary Pancreat. Dis. Int. 2017, 16, 487-492. [CrossRef]

9. Cheung, A.; Tanna, S.; Ison, M.G. Infectious complications in critically ill liver failure patients. Semin. Respir. Crit. Care Med. 2018, 39, 578-587. [CrossRef]

10. Biolatti, M.; Gugliesi, F.; Dell'Oste, V.; Landolfo, S. Modulation of the innate immune response by human cytomegalovirus. Infect. Genet. Evol. 2018, 64, 105-114. [CrossRef]

11. Chen, S.J.; Wang, S.C.; Chen, Y.C. Antiviral Agents as Therapeutic Strategies Against Cytomegalovirus Infections. Viruses 2019, 12, 21. [CrossRef] [PubMed]

12. Kornberg, A. Intravenous immunoglobulins in liver transplant patients: Perspectives of clinical immune modulation. World J. Hepatol. 2015, 7, 1494-1508. [CrossRef] [PubMed]

13. Bonaros, N.; Mayer, B.; Schachner, T.; Laufer, G.; Kocher, A. CMV-hyperimmune globulin for preventing cytomegalovirus infection and disease in solid organ transplant recipients: A meta-analysis. Clin. Transplant. 2008, 22, 89-97. [CrossRef] [PubMed]

14. Kotton, C.N.; Kumar, D.; Caliendo, A.M.; Huprikar, S.; Chou, S.; Danziger-Isakov, L.; Humar, A. The Transplantation Society International CMV Consensus Group. The Third International Consensus Guidelines on the Management of Cytomegalovirus in Solid-organ Transplantation. Transplantation 2018, 102, 900-931. [CrossRef] [PubMed] 
15. Carbone, J.; Gallego, A.; Fernandez Yañez, J.; Sousa, I.; Sarmiento, E. Potential Immunomodulatory Role of Specific Anticytomegalovirus Intravenous Immunoglobulin in Heart Recipients. Transplant. Proc. 2016, 48, 3027-3029. [CrossRef] [PubMed]

16. Van Gent, R.; Metselaar, H.J.; Kwekkeboom, J. Immunomodulation by hyperimmunoglobulins after solid organ transplantation: Beyond prevention of viral infection. Transplant. Rev. (Orlando) 2017, 31, 78-86. [CrossRef] [PubMed]

17. Carbone, J. The Immunology of Posttransplant CMV Infection: Potential Effect of CMV Immunoglobulins on Distinct Components of the Immune Response to CMV. Transplantation 2016, 100 (Suppl. 3), S11-S18. [CrossRef]

18. Jalan, R.; Pavesi, M.; Saliba, F.; Amorós, A.; Fernandez, J.; Holland-Fischer, P.; Sawhney, R.; Mookerjee, R.; Caraceni, P.; Moreau, R.; et al. The CLIF Consortium Acute Decompensation score (CLIF-C ADs) for prognosis of hospitalised cirrhotic patients without acute-on-chronic liver failure. J. Hepatol. 2015, 62, 831-840. [CrossRef]

19. Drolz, A.; Horvatits, T.; Rutter, K.; Landahl, F.; Roedl, K.; Meersseman, P.; Wilmer, A.; Kluwe, J.; Lohse, A.W.; Kluge, S.; et al. Lactate Improves Prediction of Short-Term Mortality in Critically Ill Patients With Cirrhosis: A Multinational Study. Hepatology 2019, 69, 258-269. [CrossRef]

20. Michard, B.; Artzner, T.; Lebas, B.; Besch, C.; Guillot, M.; Faitot, F.; Lefebvre, F.; Bachellie, R.P.; Castelain, V.; Maestraggi, Q.; et al. Liver transplantation in critically ill patients: Preoperative predictive factors of post-transplant mortality to avoid futility. Clin. Transplant. 2017, 31. [CrossRef]

21. Bota, D.P.; Van Nuffelen, M.; Zakariah, A.N.; Vincent, J.L. Serum levels of C-reactive protein and procalcitonin in critically ill patients with cirrhosis of the liver. J. Lab. Clin. Med. 2005, 146, 347-351. [CrossRef] [PubMed]

22. Kwekkeboom, J.; Tha-In, T.; Tra, W.M.; Hopp, W.; Boor, P.P.C.; Mancham, S.; Zondervan, P.E.; Vossen, A.C.T.M.; Kusters, J.G.; de Man, R.A.; et al. Hepatitis B immunoglobulins inhibit dendritic cells and T cells and protect against acute rejection after liver transplantation. Am. J. Transplant. 2005, 5, 2393-2402. [CrossRef] [PubMed]

23. Gerna, G.; Lilleri, D.; Fornara, C.; Bruno, F.; Gabanti, E.; Cane, I.; Furione, M.; Grazia, R.M. Differential kinetics of human cytomegalovirus load and antibody responses in primary infection of the immunocompetent and immunocompromised host. J. Gen. Virol. 2015, 96, 360-369. [CrossRef] [PubMed]

24. Hoetzenecker, K.; Hacker, S.; Hoetzenecker, W.; Sadegi, K.; Sachet, M.; Pollreisz, A.; Mangold, A.; Wliszczak, T.; Bielek, E.; Muehlbacher, F.; et al. Cytomegalovirus hyperimmunoglobulin: Mechanisms in allo-immune response in vitro. Eur. J. Clin. Investig. 2007, 37, 978-986. [CrossRef] [PubMed]

25. Farges, O.; Saliba, F.; Farhamant, H.; Samuel, D.; Bismuth, A.; Reynes, M.; Bismuth, H. Incidence of rejection and infection after liver transplantation as a function of the primary disease: Possible influence of alcohol and polyclonal immunoglobulins. Hepatology 1996, 23, 240-248. [CrossRef] [PubMed]

26. Snydman, D.R.; Werner, B.G.; Dougherty, N.N.; Griffith, J.; Rubin, R.H.; Dienstag, J.L.; Rohrer, R.H.; Freeman, R.; Jenkins, R.; Lewis, W.D.; et al. Cytomegalovirus immune globulin prophylaxis in liver transplantation. A randomized, double-blind, placebo-controlled trial. Ann. Intern. Med. 1993, 119, 984-991. [CrossRef] [PubMed]

27. Bucuvalas, J.C.; Anand, R. Studies of Pediatric Liver Transplantation Research Group.Treatment with immunoglobulin improves outcome for pediatric liver transplant recipients. Liver Transplant. 2009, 15, 1564-1569. [CrossRef]

28. Fisher, R.A.; Kistler, K.D.; Ulsh, P.; Bergman, G.E.; Morris, J. The association between cytomegalovirus immune globulin and long-term recipient and graft survival following liver transplantation. Transplant. Infect. Dis. 2012, 14, 121-131. [CrossRef]

29. Artzner, T.; Michard, B.; Besch, C.; Levesque, E.; Faitot, F. Liver transplantation for critically ill cirrhotic patients: Overview and pragmatic proposals. World J. Gastroenterol. 2018, 24, 5203-5214. [CrossRef]

30. Rausch, L.; Koenecke, C.; Koch, H.F.; Kaltenborn, A.; Emmanouilidis, N.; Pape, L.; Lehner, F.; Arelin, V.; Baumann, U.; Schrem, H. Matched-pair analysis: Identification of factors with independent influence on the development of PTLD after kidney or liver transplantation. Transplant. Res. 2016, 5, 6. [CrossRef]

31. Willuweit, K.; Bezinover, D.; Herzer, K.; Nowak, K.M.; Paul, A.; Saner, F.H. Efficacy of IgM-enriched Immunoglobulin for Vasopressor-resistant Vasoplegic Shock After Liver Transplantation. Transplantation 2019, 103, 381-386. [CrossRef] [PubMed] 
32. Tschuor, C.; Ferrarese, A.; Kuemmerli, C.; Dutkowski, P.; Burra, P.; Clavien, P.-A. Liver Allocation Study Group. Allocation of liver grafts worldwide - Is there a best system? J. Hepatol. 2019, 71, 707-718. [CrossRef] [PubMed]

33. Schlegel, A.; Linecker, M.; Kron, P.; Györi, G.; De Oliveira, M.L.; Müllhaupt, B.; Clavien, P.-A.; Dutkowski, P. Risk Assessment in High- and Low-MELD Liver Transplantation. Am. J. Transplant. 2017, 17, 1050-1063. [CrossRef] [PubMed]

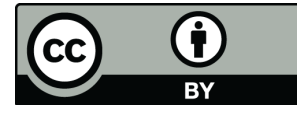

(C) 2020 by the authors. Licensee MDPI, Basel, Switzerland. This article is an open access article distributed under the terms and conditions of the Creative Commons Attribution (CC BY) license (http://creativecommons.org/licenses/by/4.0/). 